\title{
EFFECT OF INTRAVENOUS CLONIDINE AS PREMEDICATION ON HAEMODYNAMIC RESPONSE DURING LAPAROSCOPIC CHOLECYSTECTOMY
}

\author{
Vaskar Majumdar', Anghsuman Rudra Pal ${ }^{2}$
}

${ }^{1}$ Assistant Professor, Department of Anaesthesiology, AGMC, Agartala.

${ }^{2}$ Fellow, Department of Onco-Anaesthesiology, Tata Cancer Hospital, Kolkata.

\begin{abstract}
BACKGROUND

Laparoscopic approach has revolution in the surgical field, first introduced by Phillipe Mouret for laparoscopic cholecystectomy in 1987.1 During laparoscopic cholecystectomy due to pneumoperitoneum, position of patient may adversely affect the haemodynamic and ventilator parameters. During laparoscopic cholecystectomy, there were chances of haemodynamic instability and cardiovascular changes right from the pre-operative to post-operative period too. Both mechanical and neurohumoral factors, increased intra-abdominal pressure contribute to haemodynamic instability and Catecholamines mediate increase in Systemic Vascular Resistance (SVR) during PNO. It stimulates a series of hormonal and metabolic changes that constitute stress response. Clonidine is centrally acting selective partial alpha 2 adrenoceptor agonist that acts by reducing responsiveness of peripheral vessels to vasoactive substances and to sympathetic stimulation having sedative, anxiolytic, stress reducing, anaesthetic and analgesic properties.

The present study was designed to evaluate the effect of intravenous clonidine as premedication on reduction of perioperative stress response, maintaining haemodynamic stability and on modulation of postoperative pain in patients undergoing laparoscopic cholecystectomy under general anaesthesia.
\end{abstract}

\section{MATERIALS AND METHODS}

After approval from the Institutional Ethics Committee of Agartala Govt. Medical College and GBP Hospital, patient with ASA physical status I and II scheduled to undergo laparoscopic cholecystectomy from Jan. 2012 to Dec. 2012, where 100 patients are divided into two groups (Group C - have received intravenous clonidine 1.5 microgram/kg body wt. before induction and Group S have received intravenous saline before induction). Heart rate, mean arterial pressure, systolic and diastolic BP, oxygen saturation percentage and end tidal $\mathrm{CO} 2$ measured.

\section{RESULTS}

Hence mean HR, systolic BP, diastolic BP were comparable among the groups during pre-operative, after infusion of drug, after induction, 5 mins. after release of pneumoperitoneum and post-operatively 5 mins. after extubation and statistically significant difference was found at most of the time in mean HR between the two groups after intubation, $1 \mathrm{~min}, 10 \mathrm{mins}$., $20 \mathrm{mins}$., $30 \mathrm{mins}$. and 40 mins. of creating pneumoperitoneum. Post-operative analgesia by VAS scores are significantly lower in clonidine group ( $\mathrm{p}<$ 0.05) without the use of analgesic for up to 4 hours, whereas in control group with the use of analgesic VAS scores are higher.

\section{CONCLUSION}

Intravenous clonidine at $1.5 \mu \mathrm{cg} / \mathrm{kg}$ as premedication maintains heart rate, mean arterial blood pressure as it gives more haemodynamic stability, which also reduces VAS scores and prolongs the requirement of first postoperative analgesic.

\section{KEYWORDS}

Clonidine, Premedication, Haemodynamic, Laparoscopic Cholecystectomy.

HOW TO CITE THIS ARTICLE: Majumdar V, Pal AR. Effect of intravenous clonidine as premedication on haemodynamic response during laparoscopic cholecystectomy. J. Evolution Med. Dent. Sci. 2017;6(5):419-425, DOI: 10.14260/Jemds/2017/93

\section{BACKGROUND}

Phillipe Mouret introduced laparoscopic cholecystectomy in 1987.1,2 The operation table is tilted to $15 \AA$ reverse Trendelenburg position. Creation of pneumoperitoneum with $\mathrm{CO}_{2}$ and positional changes adversely affect the haemodynamic and ventilatory parameters. During laparoscopic cholecystectomy, there is reduced venous

Financial or Other, Competing Interest: None.

Submission 27-11-2016, Peer Review 01-01-2017,

Acceptance 07-01-2017, Published 16-01-2017.

Corresponding Author:

Dr. Vaskar Majumdar

Assistant Professor,

Department of Anaesthesiology,

AGMC \& GBP Hospital, Tripura-799006.

E-mail: jdebnath797@gmail.com

DOI: $10.14260 /$ jemds $/ 2017 / 93$ return; Left Ventricular End Diastolic (LVED) pressure is reduced, intrathoracic pressure is increased, right atrial and Pulmonary Artery Occlusion Pressure (PAOP) increased during insufflations. There is increase in Mean Arterial Pressures (MAP), Heart Rate (HR) And Increased Systemic Vascular Resistance (SVR) and pulmonary vascular resistance. ${ }^{3}$ Intra-abdominal Pressure (IAP) should be maintained at $6-12 \mathrm{mmHg}$, which should not be allowed to exceed $15 \mathrm{mmHg}$. After PNO, necessary changes in ventilator settings like tidal volume and respiratory rate should be done to maintain normocapnia. ${ }^{3}$

Both mechanical and neurohumoral factors contribute to haemodynamic instability, increased intra-abdominal pressure leads to release of norepinephrine, epinephrine and cortisol.4,5,6 Catecholamines mediate increase in Systemic Vascular Resistance (SVR) during PNO. 
Pain after laparoscopic cholecystectomy may be quite severe, particularly in early postoperative period either from incision or visceral manipulation and traction on nerve, vascular injury, peritoneal inflammation, presence of gas in abdomen, release of inflammatory mediators.

Clonidine is centrally acting selective partial alpha2 adrenoceptor agonist. The drug acts by reducing responsiveness of peripheral vessels to vasoactive substances and to sympathetic stimulation. Clonidine has been shown to have sedative, anxiolytic, anaesthetic and analgesic properties. It reduces the requirement of anaesthetic and analgesic agents and also attenuates the stress responses associated with anaesthesia and surgery, thus conferring haemodynamic stability. ${ }^{7.8,9}$ These characteristics suggest that clonidine may be useful in the anaesthetic management of patients undergoing laparoscopic cholecystectomy.

The present study was designed to evaluate the effect of intravenous clonidine as premedication on reduction of perioperative stress response, maintaining haemodynamic stability and on modulation of postoperative pain in patients undergoing laparoscopic cholecystectomy under general anaesthesia.

\section{Aims}

To evaluate the effects of intravenous clonidine as premedication on haemodynamic response during laparoscopic cholecystectomy.

\section{Objectives}

1. To stabilise the haemodynamic fluctuations during laparoscopic cholecystectomy using intravenous clonidine as premedication.

2. To compare the haemodynamic status of control subjects with the study subjects.

3. To evaluate the effect of intravenous clonidine on postoperative analgesic requirement.

\section{MATERIALS AND METHODS}

The present study was carried out after approval from the Institutional Ethics Committee of Agartala Govt. Medical College and GBP Hospital. A written consent form was obtained from all patients included in the study.

\section{Study Area}

GB Panth Hospital, Department of Anaesthesiology, laparoscopic surgical operation theatre.

\section{Study Population}

This study was carried out in patient with ASA physical status I and II scheduled to undergo laparoscopic cholecystectomy.

\section{Exclusion Criteria}

Patients with history of hypertension, ischaemic heart disease, terminal valvular insufficiency, AV conduction block, increased intracranial pressure, asthma, patient on MAO inhibitor, methyldopa, beta blockers, benzodiazepine was excluded from the study.

Study Period - Jan. 2012 to Dec. 2012.

Sample Size - 100 patients were divided into two groups.

\section{Study Design}

Randomised double-blind prospective study between two groups of patients scheduled to undergo laparoscopic cholecystectomy.

\section{Sample Design}

100 ASA physical status I and II, aged 18 - 60 yrs. undergoing elective laparoscopic cholecystectomy were randomly assigned to one of the two groups containing 50 patients.

Group C - Have received intravenous clonidine 1.5 microgram/kg body wt. before induction.

Group S - Have received intravenous saline before induction.

\section{Parameters to be Studied}

The following Parameters were taken into consideration for study.

\section{Demographic Variables}

a. Age in years.

b. Sex (male/female)

c. Body weight $(\mathrm{kg})$.

d. Height $(\mathrm{cm})$.

\section{Vitals}

1. Heart rate (beats per minute).

2. Mean arterial pressure ( $\mathrm{mmHg}$ by non-invasive tech).

3. Systolic and diastolic BP (mmHg by non-invasive tech).

4. Oxygen saturation percentage.

5. End tidal $\mathrm{CO} 2$.

\section{All these readings were taken at following Intervals}

- Pre-operative.

- After infusion of study drug.

- 1 min. after induction.

- 1 min. after intubation.

- 1 min. after pneumoperitoneum.

- 10 mins. after pneumoperitoneum.

- 20 mins. after pneumoperitoneum.

- 30 mins. after pneumoperitoneum.

- 40 mins. after pneumoperitoneum.

- 5 mins. after release of pneumoperitoneum.

- 5 mins. after extubation.

\section{Statistical Sample Analysis}

We calculated a sample size of 100 patients by using Software Statistical Version 6 (Tulsa, Oklahoma StatSoft Inc., 2001).

\section{Blinding Method}

The patients were unaware of which of the study drugs they received. The anaesthesiologist who was not otherwise participating in the trial prepared the study drugs into a ready-to-use form and administered to the patients.

RESULTS

\begin{tabular}{|c|c|c|c|}
\hline $\begin{array}{c}\text { Demo- } \\
\text { graphic } \\
\text { Parameter }\end{array}$ & $\begin{array}{c}\text { Group } \\
\text { Mean } \pm \text { SD }\end{array}$ & $\begin{array}{c}\text { Statistical } \\
\text { Test Results }\end{array}$ & $\begin{array}{l}\text { Statistical } \\
\text { Significance }\end{array}$ \\
\hline Age (Years) & $\begin{array}{c}\text { Group C: } 37.74 \pm 11.69 \\
\text { Group S: } 40.22 \pm 5.61\end{array}$ & $\begin{array}{c}\text { Unpaired } \\
\text { t-test } \\
\mathrm{P}=0.179\end{array}$ & $\begin{array}{c}\text { Not } \\
\text { Significant }\end{array}$ \\
\hline Height $(\mathrm{cm})$ & $\begin{array}{l}\text { Group C: } 154 \pm 8.15 \\
\text { Group S: } 153.44 \pm 6.8\end{array}$ & $\begin{array}{c}\text { Unpaired } \\
\text { t-test } \\
\mathrm{P}=0.711\end{array}$ & $\begin{array}{c}\text { Not } \\
\text { Significant }\end{array}$ \\
\hline Weight (kg) & $\begin{array}{c}\text { Group C: } 57.32 \pm 6.77 \\
\text { Group S: } 55.7 \pm 6.44\end{array}$ & $\begin{array}{c}\text { Unpaired } \\
\text { t-test } \\
\mathrm{P}=0.223\end{array}$ & $\begin{array}{c}\text { Not } \\
\text { Significant }\end{array}$ \\
\hline $\begin{array}{l}\text { Duration of } \\
\text { Surgery } \\
\text { (min.) }\end{array}$ & $\begin{array}{l}\text { Group C: } 43.96 \pm 4.08 \\
\text { Group S: } 43.42 \pm 6.94\end{array}$ & $\begin{array}{c}\text { Unpaired } \\
\text { t-test } \\
\mathrm{P}=0.647\end{array}$ & $\begin{array}{c}\text { Not } \\
\text { Significant }\end{array}$ \\
\hline $\begin{array}{l}\text { Sex (Male: } \\
\text { Female) }\end{array}$ & $\begin{array}{l}\text { Group C: } 39: 11 \\
\text { Group S: 35: } 15\end{array}$ & $\begin{array}{c}\text { Chi-square } \\
\text { test } \\
\mathrm{P}=0.3618\end{array}$ & $\begin{array}{c}\text { Not } \\
\text { Significant }\end{array}$ \\
\hline $\begin{array}{l}\text { ASA Physical } \\
\text { Status (I:II) }\end{array}$ & $\begin{array}{l}\text { Group C: } 38: 12 \\
\text { Group S: } 38: 12\end{array}$ & $\begin{array}{c}\text { Chi-square test } \\
\mathrm{P}=1.00\end{array}$ & $\begin{array}{c}\text { Not } \\
\text { Significant }\end{array}$ \\
\hline & & & \\
\hline
\end{tabular}


The above table shows that all the demographic parameters were comparable among the groups.

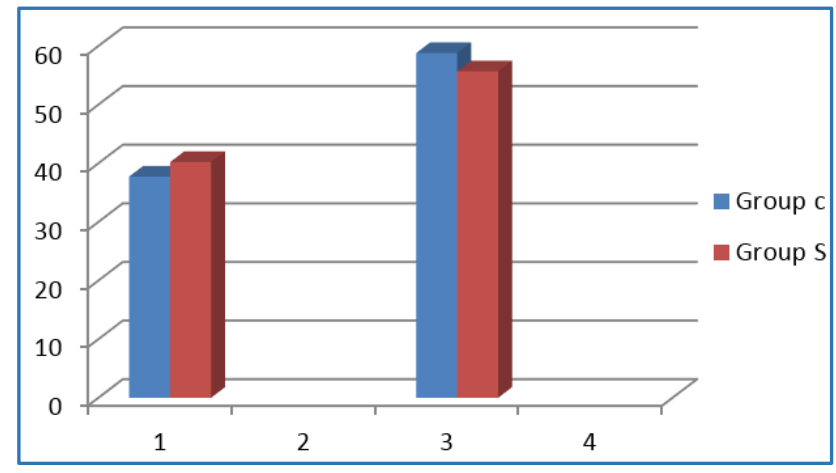

\begin{tabular}{|c|c|c|c|c|}
\hline Variables & $\begin{array}{c}\text { Group C } \\
\mathrm{N}=\mathbf{5 0} \\
\text { (mean } \pm \mathrm{SD} \text { ) }\end{array}$ & $\begin{array}{c}\text { Group S } \\
\mathrm{N}=\mathbf{5 0} \\
(\text { mean } \pm \mathrm{SD})\end{array}$ & \begin{tabular}{|l} 
Statistical \\
Test \\
Results
\end{tabular} & $\begin{array}{l}\text { Signi- } \\
\text { ficance }\end{array}$ \\
\hline $\begin{array}{l}\text { Baseline/Before } \\
\text { Premedication }\end{array}$ & $\begin{array}{c}83.26 \pm \\
9.89\end{array}$ & $\begin{array}{l}79.6 \pm \\
9.4\end{array}$ & $\begin{array}{l}\text { Unpaired } \\
\text { t-test } \\
P=0.061\end{array}$ & $\begin{array}{c}\text { Not } \\
\text { Significant }\end{array}$ \\
\hline $\begin{array}{c}\text { After Infusion } \\
\text { of Study Drug/ } \\
\text { Saline }\end{array}$ & $\begin{array}{c}83.22 \pm \\
11.54\end{array}$ & $\begin{array}{c}80.44 \pm \\
11.97\end{array}$ & $\begin{array}{l}\text { Unpaired } \\
\text { t-test } \\
\mathrm{P}=0.240\end{array}$ & $\begin{array}{c}\text { Not } \\
\text { Significant }\end{array}$ \\
\hline $\begin{array}{c}\text { After } \\
\text { Induction }\end{array}$ & $\begin{array}{c}82.44 \pm \\
12.54\end{array}$ & $\begin{array}{c}78.78 \pm \\
10.90\end{array}$ & $\begin{array}{l}\text { Unpaired } \\
\mathrm{t} \text {-test } \\
\mathrm{P}=0.116\end{array}$ & $\begin{array}{c}\text { Not } \\
\text { Significant }\end{array}$ \\
\hline $\begin{array}{l}1 \text { min After } \\
\text { Intubation }\end{array}$ & $\begin{array}{c}86.92 \pm \\
12.46\end{array}$ & $\begin{array}{l}91.96 \pm \\
12.36\end{array}$ & $\begin{array}{l}\text { Unpaired } \\
\text { t-test } \\
\mathrm{P}=0.045\end{array}$ & Significant \\
\hline $\begin{array}{l}1 \text { min After } \\
\text { Pneumo- } \\
\text { peritoneum }\end{array}$ & $\begin{array}{c}89.64 \pm \\
15.69\end{array}$ & $\begin{array}{l}96.12 \pm \\
13.80\end{array}$ & $\begin{array}{l}\text { Unpaired } \\
\text { t-test } \\
\mathrm{P}=0.031\end{array}$ & Significant \\
\hline $\begin{array}{l}10 \text { mins. After } \\
\text { Pneumo- } \\
\text { peritoneum }\end{array}$ & $\begin{array}{c}89.24 \pm \\
14.19\end{array}$ & $\begin{array}{c}96.94 \pm \\
13.00\end{array}$ & $\begin{array}{l}\text { Unpaired } \\
\text { t-test } \\
\mathrm{P}=0.006\end{array}$ & Significant \\
\hline $\begin{array}{l}20 \text { mins. After } \\
\text { Pneumo- } \\
\text { peritoneum }\end{array}$ & $\begin{array}{c}90.34 \pm \\
15.10\end{array}$ & $\begin{array}{l}98.08 \pm \\
13.46\end{array}$ & $\begin{array}{l}\text { Unpaired } \\
\text { t-test } \\
\mathrm{P}=0.008\end{array}$ & Significant \\
\hline $\begin{array}{l}30 \text { mins. After } \\
\text { Pneumo- } \\
\text { peritoneum }\end{array}$ & $\begin{array}{c}88.72 \pm \\
14.51\end{array}$ & $\begin{array}{l}94.7 \pm \\
11.42\end{array}$ & $\begin{array}{l}\text { Unpaired } \\
\text { t-test } \\
\mathrm{P}=0.024\end{array}$ & Significant \\
\hline $\begin{array}{l}40 \text { mins. After } \\
\text { Pneumo- } \\
\text { peritoneum }\end{array}$ & $\begin{array}{c}87.38 \pm \\
13.05\end{array}$ & $\begin{array}{c}94.86 \pm \\
10.99\end{array}$ & $\begin{array}{l}\text { Unpaired } \\
\text { t-test } \\
\mathrm{P}=0.003\end{array}$ & Significant \\
\hline $\begin{array}{c}5 \text { mins. After } \\
\text { Pneumo- } \\
\text { peritoneum Release }\end{array}$ & $\begin{array}{c}86.16 \pm \\
12.29\end{array}$ & $\begin{array}{l}90.30 \pm \\
10.50\end{array}$ & $\begin{array}{l}\text { Unpaired } \\
\text { t-test } \\
\mathrm{P}=0.073\end{array}$ & $\begin{array}{c}\text { Not } \\
\text { Significant }\end{array}$ \\
\hline $\begin{array}{c}5 \text { mins. After } \\
\text { Extubation (P.O.) }\end{array}$ & $\begin{array}{c}81.24 \pm \\
8.19\end{array}$ & $\begin{array}{c}81.00 \pm \\
6.00\end{array}$ & $\begin{array}{l}\text { Unpaired } \\
\mathrm{t} \text {-test } \\
\mathrm{P}=0.868\end{array}$ & $\begin{array}{c}\text { Not } \\
\text { Significant }\end{array}$ \\
\hline \multicolumn{5}{|c|}{$\begin{array}{l}\text { Table 2. Table comparing Heart Rate in } \\
\text { (Beats/Minute) among the Study Groups at } \\
\text { Specified Points of Time during Surgery }(N=100)\end{array}$} \\
\hline
\end{tabular}

Applying the 2-tailed unpaired t-test (assuming equal variance) between the mean of HR of each group $p<0.05$ was found at after intubation 1, 10, 20, 30, 40 mins. after creating pneumoperitoneum and $\mathrm{p}>0.05$ was found preoperatively, after infusion of clonidine or normal saline after induction of 5 mins. after release of pneumoperitoneum and postoperatively 5 mins. after extubation. Hence, mean HR were comparable among the groups during pre-operative after infusion of drug, after induction, 5 mins. after release of pneumoperitoneum and postoperatively 5 mins. after extubation and statistically significant difference was found at most of the time in mean HR between the two groups after intubation, 1 min., 10 mins., 20 mins., 30 mins., 40 mins. of creating pneumoperitoneum.

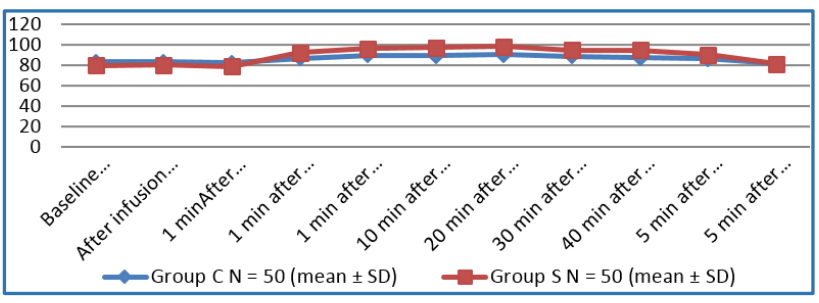

\begin{tabular}{|c|c|c|c|c|}
\hline Variables & $\begin{array}{c}\text { Group C } \\
\text { N }=50 \\
\text { (Mean } \pm \\
\text { SD) }\end{array}$ & $\begin{array}{c}\text { Group S } \\
\mathrm{N}=\mathbf{5 0} \\
\text { (Mean } \pm \\
\text { SD) }\end{array}$ & $\begin{array}{c}\text { Statistical } \\
\text { Test } \\
\text { Results }\end{array}$ & Significance \\
\hline $\begin{array}{l}\text { Baseline/before } \\
\text { premedication }\end{array}$ & $\begin{array}{c}125.42 \pm \\
11.66\end{array}$ & $\begin{array}{c}122.30 \pm \\
7.27\end{array}$ & $\begin{array}{c}\text { Unpaired } \\
\text { t-test } \\
\mathrm{P}=0.112\end{array}$ & $\begin{array}{c}\text { Not } \\
\text { Significant }\end{array}$ \\
\hline $\begin{array}{c}\text { After Infusion of } \\
\text { Study } \\
\text { Drug/Saline }\end{array}$ & $\begin{array}{c}119.26 \pm \\
13.48\end{array}$ & $\begin{array}{c}122.10 \pm \\
7.30\end{array}$ & $\begin{array}{c}\text { Unpaired } \\
\text { t-test } \\
\mathrm{P}=0.193\end{array}$ & $\begin{array}{c}\text { Not } \\
\text { Significant }\end{array}$ \\
\hline $\begin{array}{c}\text { After } \\
\text { Induction }\end{array}$ & $\begin{array}{c}117.36 \pm \\
14.06\end{array}$ & $\begin{array}{c}118.02 \pm \\
9.93\end{array}$ & $\begin{array}{c}\text { Unpaired } \\
\text { t-test } \\
\mathrm{P}=0.787\end{array}$ & $\begin{array}{c}\text { Not } \\
\text { Significant }\end{array}$ \\
\hline $\begin{array}{l}1 \text { min After } \\
\text { Intubation }\end{array}$ & $\begin{array}{c}132.62 \pm \\
15.59\end{array}$ & $\begin{array}{c}139.16 \pm \\
14.85\end{array}$ & $\begin{array}{c}\text { Unpaired } \\
\text { t-test } \\
\mathrm{P}=0.014\end{array}$ & Significant \\
\hline $\begin{array}{c}1 \text { min after } \\
\text { Pneumo- } \\
\text { peritoneum }\end{array}$ & $\begin{array}{c}132.16 \pm \\
14.84\end{array}$ & $\begin{array}{c}147.92 \pm \\
12.54\end{array}$ & $\begin{array}{c}\text { Unpaired } \\
\text { t-test } \\
\mathrm{P}=0.000 \\
\end{array}$ & Significant \\
\hline $\begin{array}{c}10 \text { mins. After } \\
\text { Pneumo- } \\
\text { peritoneum }\end{array}$ & $\begin{array}{c}135.22 \pm \\
12.58\end{array}$ & $\begin{array}{c}148.38 \pm \\
13.12\end{array}$ & $\begin{array}{c}\text { Unpaired } \\
\text { t-test } \\
\mathrm{P}=0.000\end{array}$ & Significant \\
\hline $\begin{array}{c}20 \text { mins. After } \\
\text { Pneumo- } \\
\text { peritoneum }\end{array}$ & $\begin{array}{c}136.02 \pm \\
12.84\end{array}$ & $\begin{array}{c}148.96 \pm \\
13.34\end{array}$ & $\begin{array}{c}\text { Unpaired } \\
\text { t-test } \\
\mathrm{P}=0.000\end{array}$ & Significant \\
\hline $\begin{array}{c}30 \text { mins. After } \\
\text { Pneumo- } \\
\text { peritoneum }\end{array}$ & $\begin{array}{c}134.02 \pm \\
13.75\end{array}$ & $\begin{array}{c}143.38 \pm \\
9.80\end{array}$ & $\begin{array}{c}\text { Unpaired } \\
\text { t-test } \\
\mathrm{P}=0.000\end{array}$ & Significant \\
\hline $\begin{array}{l}40 \text { mins. After } \\
\text { Pneumo- } \\
\text { peritoneum }\end{array}$ & $\begin{array}{c}133.98 \pm \\
13.87\end{array}$ & $\begin{array}{c}139.30 \pm \\
8.37\end{array}$ & $\begin{array}{l}\begin{array}{c}\text { Unpaired } \mathrm{t}- \\
\text { test }\end{array} \\
\mathrm{P}=0.022\end{array}$ & Significant \\
\hline $\begin{array}{l}5 \text { mins. After } \\
\text { Pneumo- } \\
\text { peritoneum } \\
\text { Release }\end{array}$ & $\begin{array}{c}130.86 \pm \\
11.76\end{array}$ & $\begin{array}{c}135.16 \pm \\
7.62\end{array}$ & $\begin{array}{c}\text { Unpaired } \\
\text { t-test } \\
\mathrm{P}=0.032\end{array}$ & Significant \\
\hline $\begin{array}{c}5 \text { mins. After } \\
\text { Extubation (P.O.) }\end{array}$ & $\begin{array}{c}123.50 \pm \\
5.23\end{array}$ & $\begin{array}{c}124.96 \pm \\
3.02\end{array}$ & $\begin{array}{c}\text { Unpaired } \\
\text { t-test } \\
\mathrm{P}=0.091\end{array}$ & $\begin{array}{c}\text { Not } \\
\text { Significant }\end{array}$ \\
\hline \multicolumn{5}{|c|}{$\begin{array}{c}\text { Table 3. Table comparing Systolic Blood } \\
\text { Pressure (mmHg) among the Study Groups at } \\
\text { Specified Points of Time during Surgery }(N=100)\end{array}$} \\
\hline
\end{tabular}

Applying the 2-tailed unpaired t-test (assuming equal variance) between the mean of SBP of each group, $p<0.05$ was found after intubation, $1 \mathrm{~min}$. after pneumoperitoneum, 10 mins. after pneumoperitoneum, 20 mins. after pneumoperitoneum, 30 mins. after pneumoperitoneum, 40 mins. after pneumoperitoneum and 5 mins. after release of 
pneumoperitoneum and $\mathrm{p}>0.05$ was found preoperatively, after infusion of drug, after induction and postoperatively 5 mins. after extubation. Hence, during preoperative after infusion of drug, after induction and postoperative mean SBP were comparable among the groups and statistically significant difference was found at most of the time in mean SBP between the two groups after intubation, during various stages of pneumoperitoneum and 5 mins. after release of pneumoperitoneum.

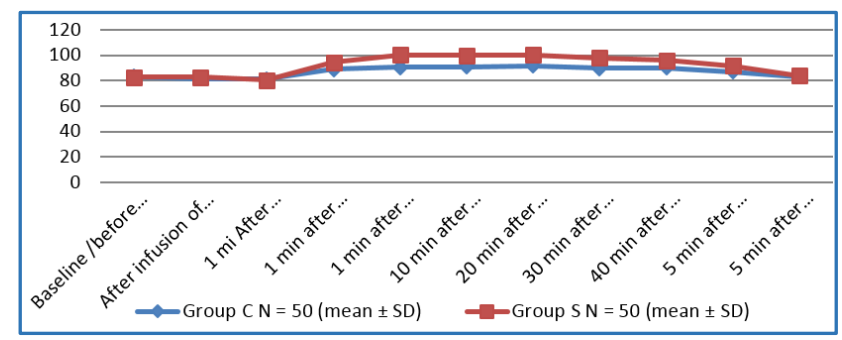

\begin{tabular}{|c|c|c|c|c|}
\hline Variables & $\begin{array}{c}\text { Group } \\
C \\
N=50 \\
(\text { mean } \\
\pm \text { SD) }\end{array}$ & $\begin{array}{c}\text { Group S } \\
\mathrm{N}=5 \mathbf{5 0} \\
\text { (mean } \\
\pm \mathrm{SD})\end{array}$ & $\begin{array}{c}\text { Statistical } \\
\text { Test } \\
\text { Results }\end{array}$ & Significance \\
\hline $\begin{array}{l}\text { Baseline/Before } \\
\text { Premedication }\end{array}$ & $\begin{array}{c}83.06 \pm \\
6.74\end{array}$ & $\begin{array}{c}82.90 \pm \\
5.70\end{array}$ & $\begin{array}{c}\text { Unpaired } \\
\text { t-test } \\
\mathrm{P}=0.899\end{array}$ & $\begin{array}{c}\text { Not } \\
\text { Significant }\end{array}$ \\
\hline $\begin{array}{c}\text { After Infusion of } \\
\text { Study } \\
\text { Drug/Saline } \\
\end{array}$ & $\begin{array}{c}81.66 \pm \\
10.11\end{array}$ & $\begin{array}{c}82.74 \pm \\
5.41\end{array}$ & $\begin{array}{c}\text { Unpaired } \\
\text { t-test } \\
\mathrm{P}=0.507 \\
\end{array}$ & $\begin{array}{c}\text { Not } \\
\text { Significant }\end{array}$ \\
\hline $\begin{array}{c}\text { After } \\
\text { Induction }\end{array}$ & $\begin{array}{l}81.18 \pm \\
12.03\end{array}$ & $\begin{array}{c}80.32 \pm \\
6.50\end{array}$ & $\begin{array}{c}\begin{array}{c}\text { Unpaired } t- \\
\text { test }\end{array} \\
\mathrm{P}=0.658\end{array}$ & $\begin{array}{c}\text { Not } \\
\text { Significant }\end{array}$ \\
\hline $\begin{array}{l}1 \text { min After } \\
\text { Intubation }\end{array}$ & $\begin{array}{l}89.12 \pm \\
8.86\end{array}$ & $\begin{array}{c}94.56 \pm \\
5.41\end{array}$ & $\begin{array}{c}\text { Unpaired } \\
\text { t-test } \\
\mathrm{P}=0.001 \\
\end{array}$ & $\begin{array}{c}\text { Highly } \\
\text { Significant }\end{array}$ \\
\hline $\begin{array}{l}1 \text { min. after } \\
\text { Pneumo- } \\
\text { peritoneum }\end{array}$ & $\begin{array}{c}90.36 \pm \\
11.40\end{array}$ & $\begin{array}{c}100.46 \pm \\
7.70\end{array}$ & $\begin{array}{l}\text { Unpaired } \\
\text { t-test } \\
\mathrm{P}=0.000\end{array}$ & $\begin{array}{l}\text { Highly } \\
\text { Significant }\end{array}$ \\
\hline $\begin{array}{l}10 \text { mins. After } \\
\text { Pneumo- } \\
\text { peritoneum }\end{array}$ & $\begin{array}{c}90.88 \pm \\
9.38\end{array}$ & $\begin{array}{c}99.96 \pm \\
8.99\end{array}$ & $\begin{array}{c}\text { Unpaired } \\
\text { t-test } \\
\mathrm{P}=0.000\end{array}$ & $\begin{array}{c}\text { Highly } \\
\text { Significant }\end{array}$ \\
\hline $\begin{array}{c}20 \text { mins. after } \\
\text { Pneumo- } \\
\text { peritoneum }\end{array}$ & $\begin{array}{c}91.72 \pm \\
8.30\end{array}$ & $\begin{array}{c}100.22 \pm \\
6.56\end{array}$ & $\begin{array}{c}\text { Unpaired } \\
\text { t-test } \\
\mathrm{P}=0.000\end{array}$ & $\begin{array}{c}\text { Highly } \\
\text { Significant }\end{array}$ \\
\hline $\begin{array}{l}30 \text { mins. After } \\
\text { Pneumo- } \\
\text { peritoneum }\end{array}$ & $\begin{array}{c}89.86 \pm \\
8.77\end{array}$ & $\begin{array}{c}97.88 \pm \\
6.39\end{array}$ & $\begin{array}{c}\text { Unpaired } \\
\text { t-test } \\
\mathrm{P}=0.000\end{array}$ & $\begin{array}{l}\text { Highly } \\
\text { Significant }\end{array}$ \\
\hline $\begin{array}{l}40 \text { mins. After } \\
\text { Pneumo- } \\
\text { peritoneum }\end{array}$ & $\begin{array}{c}90.20 \pm \\
8.76\end{array}$ & $\begin{array}{c}95.86 \pm \\
5.68\end{array}$ & $\begin{array}{c}\text { Unpaired } \\
\text { t-test } \\
\mathrm{P}=0.000 \\
\end{array}$ & $\begin{array}{c}\text { Highly } \\
\text { Significant }\end{array}$ \\
\hline $\begin{array}{l}5 \text { mins. After } \\
\text { Pneumo- } \\
\text { peritoneum } \\
\text { Release }\end{array}$ & $\begin{array}{c}86.98 \pm \\
7.09\end{array}$ & $\begin{array}{c}91.62 \pm \\
6.36\end{array}$ & $\begin{array}{l}\text { Unpaired } \\
\text { t-test } \\
\mathrm{P}=0.001\end{array}$ & Significant \\
\hline $\begin{array}{c}5 \text { mins. After } \\
\text { Extubation (P.O.) }\end{array}$ & $\begin{array}{c}83.08 \pm \\
3.54\end{array}$ & $\begin{array}{c}84.94 \pm \\
3.18\end{array}$ & $\begin{array}{c}\text { Unpaired } \\
\text { t-test } \\
\mathrm{P}=0.006\end{array}$ & Significant \\
\hline \multicolumn{5}{|c|}{$\begin{array}{l}\text { Table 4. Table comparing Diastolic Blood } \\
\text { Pressure ( }(\mathrm{mmHg}) \text { among the Study Groups } \\
\text { at Specified Points of Time during Surgery }(\mathrm{N}=100)\end{array}$} \\
\hline
\end{tabular}

Applying the 2-tailed unpaired t-test (assuming equal variance) between the mean of DBP of each group, $p<0.05$ was found at after intubation, during different time intervals after creating pneumoperitoneum, 5 mins. after release of pneumoperitoneum and postoperatively 5 mins. after extubation and $\mathrm{p}>0.05$ was found preoperatively, after infusion of drug and after induction. Hence, during preoperative, after infusion of drug and after induction mean DBP were comparable among the groups and statistical significance was found at most of the time in mean DBP between the two groups after intubation, during different time intervals after creating pneumoperitoneum, 5 mins. after release of pneumoperitoneum and postoperatively 5 mins. after extubation.

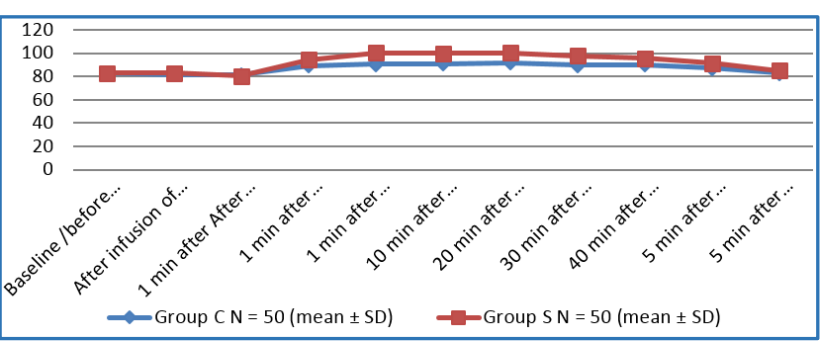

\begin{tabular}{|c|c|c|c|c|}
\hline Variables & $\begin{array}{c}\text { Group C } \\
\mathrm{N}=\mathbf{5 0} \\
(\mathrm{mean} \pm \\
\mathrm{SD})\end{array}$ & \begin{tabular}{|c|} 
Group S \\
$\mathrm{N}=\mathbf{5 0}$ \\
$(\mathrm{mean} \pm$ \\
$\mathrm{SD})$
\end{tabular} & $\begin{array}{c}\text { Statistical } \\
\text { Test } \\
\text { Results }\end{array}$ & Significance \\
\hline $\begin{array}{l}\text { Baseline/before } \\
\text { Premedication }\end{array}$ & $\begin{array}{c}97.08 \pm \\
7.86\end{array}$ & $\begin{array}{c}96.02 \pm \\
5.80\end{array}$ & $\begin{array}{c}\text { Unpaired } \\
\text { t-test } \\
\mathrm{P}=0.445\end{array}$ & $\begin{array}{c}\text { Not } \\
\text { Significant }\end{array}$ \\
\hline $\begin{array}{l}\text { After infusion of } \\
\text { Study Drug/Saline }\end{array}$ & $\begin{array}{c}93.66 \pm \\
8.59\end{array}$ & $\begin{array}{c}95.86 \pm \\
5.40\end{array}$ & $\begin{array}{c}\text { Unpaired } \\
\text { t-test } \\
\mathrm{P}=0.129 \\
\end{array}$ & $\begin{array}{c}\text { Not } \\
\text { Significant }\end{array}$ \\
\hline $\begin{array}{c}\text { After } \\
\text { Induction }\end{array}$ & $\begin{array}{l}93.62 \pm \\
11.62\end{array}$ & $\begin{array}{c}93.02 \pm \\
7.38\end{array}$ & $\begin{array}{c}\text { Unpaired } \\
\text { t-test } \\
\mathrm{P}=0.759\end{array}$ & $\begin{array}{c}\text { Not } \\
\text { Significant }\end{array}$ \\
\hline $\begin{array}{l}1 \text { min. after } \\
\text { Intubation }\end{array}$ & $\begin{array}{c}103.26 \pm \\
10.22\end{array}$ & $\begin{array}{c}108.90 \pm \\
7.57\end{array}$ & $\begin{array}{c}\text { Unpaired } \\
\text { t-test } \\
\mathrm{P}=0.002\end{array}$ & Significant \\
\hline $\begin{array}{c}1 \text { min. after } \\
\text { Pneumo- } \\
\text { peritoneum }\end{array}$ & $\begin{array}{c}104.76 \pm \\
11.78\end{array}$ & $\begin{array}{c}116.12 \pm \\
9.08\end{array}$ & $\begin{array}{c}\text { Unpaired } \\
\text { t-test } \\
\mathrm{P}=0.000 \\
\end{array}$ & Significant \\
\hline $\begin{array}{c}10 \text { mins. after } \\
\text { Pneumo- } \\
\text { peritoneum }\end{array}$ & $\begin{array}{c}105.64 \pm \\
9.94\end{array}$ & $\begin{array}{c}115.98 \pm \\
9.79\end{array}$ & $\begin{array}{c}\text { Unpaired } \\
\text { t-test } \\
\mathrm{P}=0.000\end{array}$ & Significant \\
\hline $\begin{array}{c}20 \text { mins. after } \\
\text { Pneumo- } \\
\text { peritoneum }\end{array}$ & $\begin{array}{c}106.96 \pm \\
9.53\end{array}$ & $\begin{array}{c}116.40 \pm \\
8.05\end{array}$ & \begin{tabular}{|c|} 
Unpaired \\
t-test \\
$\mathrm{P}=0.000$ \\
\end{tabular} & Significant \\
\hline $\begin{array}{l}30 \text { mins. after } \\
\text { Pneumo- } \\
\text { peritoneum }\end{array}$ & $\begin{array}{c}104.40 \pm \\
9.92\end{array}$ & $\begin{array}{c}113.08 \pm \\
7.13\end{array}$ & $\begin{array}{c}\text { Unpaired } \\
\text { t-test } \\
\mathrm{P}=0.000\end{array}$ & Significant \\
\hline $\begin{array}{c}40 \text { min after } \\
\text { Pneumo- } \\
\text { peritoneum }\end{array}$ & $\begin{array}{c}104.66 \pm \\
9.88\end{array}$ & $\begin{array}{c}110.18 \pm \\
5.62\end{array}$ & $\begin{array}{c}\text { Unpaired } \\
t \text {-test } \\
\mathrm{P}=0.001 \\
\end{array}$ & Significant \\
\hline $\begin{array}{l}5 \text { mins. after } \\
\text { Pneumo- } \\
\text { peritoneum } \\
\text { Release } \\
\end{array}$ & $\begin{array}{c}101.24 \pm \\
9.86\end{array}$ & $\begin{array}{c}105.96 \pm \\
6.09\end{array}$ & $\begin{array}{l}\text { Unpaired } \\
\text { t-test } \\
\mathrm{P}=0.001\end{array}$ & Significant \\
\hline $\begin{array}{l}5 \text { mins. after } \\
\text { Extubation }\end{array}$ & $\begin{array}{c}96.50 \pm \\
3.86\end{array}$ & $\begin{array}{c}98.32 \pm \\
2.82\end{array}$ & $\begin{array}{c}\text { Unpaired } \\
\text { t-test } \\
\mathrm{P}=0.008\end{array}$ & Significant \\
\hline \multicolumn{5}{|c|}{$\begin{array}{c}\text { Table 5. Table comparing Mean Arterial Pressure } \\
\text { (mmHg) among the Study Groups at Specified } \\
\text { Points of Time During Surgery }(N=100)\end{array}$} \\
\hline
\end{tabular}


Applying the 2-tailed unpaired t-test (assuming equal variance) between the mean of MAP of each group $p<0.05$ was found at after intubation, during different time intervals after creating pneumoperitoneum, 5 mins. after release of pneumoperitoneum and postoperatively 5 mins. after extubation and $\mathrm{p}>0.05$ was found preoperative, after infusion of drug and after induction. Hence, during preoperative, after infusion of drug, after induction and MAP were comparable among the groups and statistically significance was found at most of the time in mean MAP between the two groups after intubation, during different time intervals after creating pneumoperitoneum and 5 mins. after release of pneumoperitoneum and postoperatively 5 mins. after extubation.

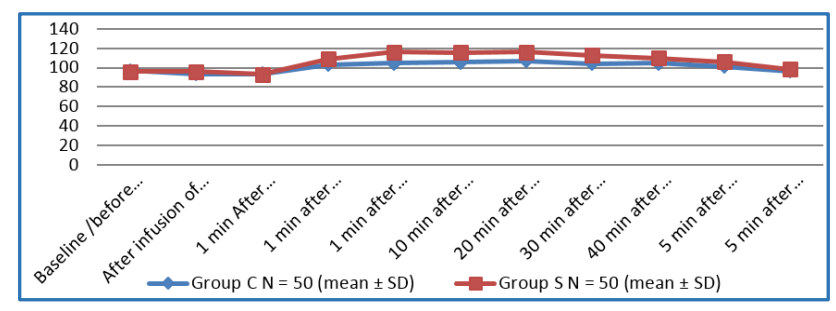

\begin{tabular}{|c|c|c|c|c|}
\hline $\begin{array}{c}\text { Time } \\
\text { Interval }\end{array}$ & $\begin{array}{c}\text { Group C } \\
\mathrm{N}=\mathbf{5 0} \\
(\mathrm{mean} \pm \mathrm{SD})\end{array}$ & $\begin{array}{c}\text { Group S } \\
\mathrm{N}=\mathbf{5 0} \\
\text { (mean } \pm \text { SD) }\end{array}$ & $\begin{array}{c}\text { Statistical } \\
\text { Test } \\
\text { Results }\end{array}$ & $\begin{array}{l}\text { Signifi- } \\
\text { cance }\end{array}$ \\
\hline \begin{tabular}{|l|}
5 mins. \\
to 2 hrs. \\
\end{tabular} & $\begin{array}{c}3.00 \pm \\
00 \\
\end{array}$ & $\begin{array}{l}5.12 \pm \\
0.799 \\
\end{array}$ & $\begin{array}{c}\text { Unpaired t-test } \\
\mathrm{P}=0.000\end{array}$ & $\begin{array}{c}\text { Highly } \\
\text { significant }\end{array}$ \\
\hline $\begin{array}{c}2 \mathrm{hrs} . \\
\text { to } 4 \mathrm{hrs} .\end{array}$ & $\begin{array}{l}3.46 \pm \\
0.503\end{array}$ & $\begin{array}{l}3.76 \pm \\
0.431\end{array}$ & $\begin{array}{c}\text { Unpaired } t \text {-test } \\
P=0.002\end{array}$ & Significant \\
\hline $\begin{array}{c}4 \mathrm{hrs} . \\
\text { to } 8 \mathrm{hrs} .\end{array}$ & $\begin{array}{l}3.92 \pm \\
0.724\end{array}$ & $\begin{array}{l}4.96 \pm \\
0.880\end{array}$ & $\begin{array}{c}\text { Unpaired } t \text {-test } \\
P=0.000\end{array}$ & $\begin{array}{c}\text { Highly } \\
\text { Significant }\end{array}$ \\
\hline $\begin{array}{c}8 \text { hrs. } \\
\text { to } 12 \\
\text { hrs. }\end{array}$ & $\begin{array}{l}3.40 \pm \\
0.495\end{array}$ & $\begin{array}{l}3.46 \pm \\
0.503\end{array}$ & $\begin{array}{c}\text { Unpaired t-test } \\
\mathrm{P}=0.549\end{array}$ & $\begin{array}{c}\text { Not } \\
\text { Significant }\end{array}$ \\
\hline
\end{tabular}

The table shows that VAS scores are significantly lower in clonidine group ( $<<0.05$ ) without the use of analgesic for up to 4 hours, whereas in control group with the use of analgesic VAS scores are higher.

\begin{tabular}{|c|c|c|c|c|c|}
\hline \multirow{2}{*}{$\begin{array}{c}\text { Time } \\
\text { Inter- } \\
\text { val }\end{array}$} & \multicolumn{2}{|c|}{$\begin{array}{l}\text { Group C } \\
(N=50)\end{array}$} & \multicolumn{2}{|c|}{$\begin{array}{l}\text { Group } S \\
(N=50)\end{array}$} & $\begin{array}{l}\text { Statisti- } \\
\text { cal Test } \\
\text { Results }\end{array}$ \\
\hline & Number & $\%$ & Number & $\%$ & \multirow{4}{*}{$\begin{array}{c}\text { Chi- } \\
\text { square }= \\
57.44 \\
\text { DF = 2 } \\
\text { P }<0.001\end{array}$} \\
\hline $\begin{array}{l}5 \mathrm{~min} \text {. } \\
\text { to } 2 \mathrm{hrs} \text {. }\end{array}$ & 0 & $00 \%$ & 100 & $100 \%$ & \\
\hline $\begin{array}{l}2 \mathrm{hrs} . \\
\text { to } 4 \mathrm{hrs} \text {. }\end{array}$ & 6 & $12 \%$ & 29 & $38 \%$ & \\
\hline $\begin{array}{l}4 \mathrm{hrs} . \\
\text { to } 8 \mathrm{hrs} \text {. }\end{array}$ & 31 & $62 \%$ & 36 & $72 \%$ & \\
\hline & $\begin{array}{r}\text { Table } 7.1 \\
\text { Analg }\end{array}$ & 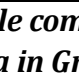 & o C and & up $S$ & \\
\hline
\end{tabular}

Requirement of first dose of analgesic was prolonged in clonidine group for up to $4 \mathrm{hrs}$. post-operatively ( $\mathrm{p}<0.001$ ). Inj. Diclofenac sodium was used as rescue analgesic in both the groups.

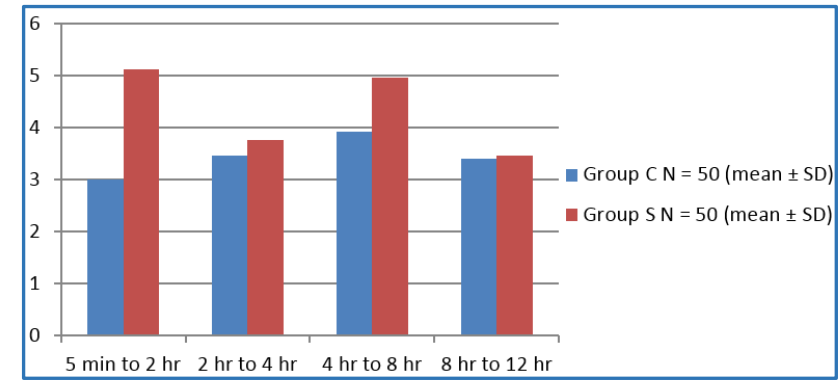

\section{DISCUSSION}

Laparoscopic cholecystectomy is the gold standard surgical procedure for treatment of chronic cholecystitis. Laparoscopic surgery has proved immensely as a standard surgical procedure in gynaecological and surgical fields. To reduce the incidence and severity of these potentially hazardous changes in haemodynamic status during laparoscopy, several interventions have been tried. These interventions include fluid loading or tilting the patient slight head-down position before peritoneal insufflations, use of compression device in lower extremities, use of vasodilating anaesthetic agent like isoflurane or other vasodilating substance like nitroglycerin and nicardipine and use of $\alpha_{2}$ adrenergic agonists like clonidine (or dexmedetomidine and $\beta$-blocking agents and remifentanil alternative techniques of CO2 pneumoperitoneum such as use of inert gases like helium, argon or gasless laparoscopy.

Clonidine, an imidazoline derivative is a selective $\alpha_{2}$ adrenergic agonist. It is a potent antihypertensive drug. It produces a fall in the heart rate and blood pressure associated with decreased SVR and cardiac output. Studies using oral clonidine $(150 \mu \mathrm{g})$ administered 60 to 90 mins. before induction of anaesthesia have shown promising results in minimising the haemodynamic changes during laparoscopic surgery. Although, the bioavailability of clonidine after oral administration is over $90 \%$, it requires 2 to $4 \mathrm{~h}$ to develop its peak effect necessitating its ingestion at least $2 \mathrm{~h}$ prior to induction to have clinically desirable actions. As intravenous clonidine has an onset of action within 15 mins. (with a peak at 30 mins.), its administration half an hour before induction of anaesthesia is a better option.

In this perspective, a randomised, double-blind, prospective, placebo-controlled study was conducted at Agartala Govt. Medical College and GBP Hospital, Agartala in which 100 patients were randomly allocated into 2 groups. One group (Group C) received clonidine intravenously and the other group (Group S) received normal saline intravenously. The haemodynamic changes during perioperative period were recorded.

A study ${ }^{10,11}$ reported significant rise in MAP in Group P (Placebo) as compared to Group C (clonidine $150 \mathrm{mcg}$ ) after intubation (113.56 $\pm 16.33 \mathrm{mmHg}$ vs $93.70 \pm 7.33$; p .001), after PNO at 15 mins. as $(114.13 \pm 16.57 \mathrm{mmHg}$ vs $93.83 \pm 8.10$; $\mathrm{p}<$ $0.001)$, at 30 mins. $(108.60 \pm 15.11 \mathrm{mmHg}$ vs $93.64 \pm 8.40 ; \mathrm{p}=$ 0.033). In our study, similar significant rise in MAP was noted in Group S as compared to Group C at intubation (108.90 \pm $7.57 \mathrm{mmHg}$ vs $103.26 \pm 10.22 \mathrm{mmHg} ; \mathrm{p}=0.002$ ) after PNO at 10 mins. (115.98 $\pm 9.79 \mathrm{mmHg}$ vs $105.64 \pm 9.94 \mathrm{mmHg}$; $<$ $0.001)$, at 20 mins. $(116.40 \pm 8.05 \mathrm{mmHg}$ vs $106.96 \pm 9.53$ mmHg; $\mathrm{p}<0.001)$ and at 30 mins. $(113.08 \pm 7.13 \mathrm{mmHg}$ vs $104.40 \pm 9.92 \mathrm{mmHg} ; \mathrm{p}<0.001)$. 
The study also reported significant rise in HR in Group P compared to Group C after intubation $(107.76 \pm 14.06 \mathrm{bpm}$ vs $87.26 \pm 11.34$ bpm; $p=0.006$ ), after PNO at 15 mins. as (96.06 $\pm 21.81 \mathrm{bpm}$ vs $75.76 \pm 10.07 \mathrm{bpm} ; \mathrm{p}=0.008)$, at 30 mins. (94.76 $\pm 19.79 \mathrm{bpm}$ vs $75.70 \pm 10.20 \mathrm{bpm} ; \mathrm{p}=0.004)$, which were comparable to present study that is at intubation (91.96 $\pm 12.36 \mathrm{bpm}$ vs $86.92 \pm 12.46 \mathrm{bpm}$; $\mathrm{P}=0.045$ ), after PNO at 15 mins. $(96.94 \pm 13.00 \mathrm{bpm}$ vs $89.24 \pm 14.19 \mathrm{bpm} ; \mathrm{P}=$ $0.006)$, at 20 mins. $(98.08 \pm 13.46 \mathrm{bpm}$ vs $90.34 \pm 15.10 \mathrm{bpm}$; $\mathrm{P}=0.008)$, and at 30 mins. $(94.7 \pm 11.42 \mathrm{bpm}$ vs $88.72 \pm 14.51$ bpm; $\mathrm{P}=0.024$ ).

In the same study, significant rise in HR was observed in control group compared to clonidine group (before PNO; 70 bpm vs $62 \mathrm{bpm}$ ), at (15 mins. after PNO; $82 \mathrm{bpm}$ vs $70 \mathrm{bpm}$ ) and at (30 mins.; 83 bpm vs $78 \mathrm{bpm})(\mathrm{p}<0.001)$. In our study also mean HR was significantly raised in Group $S$, that is before PNO $(91.96 \pm 12.36 \mathrm{bpm}$ vs $86.92 \pm 12.46 \mathrm{bpm}$; $\mathrm{P}=$ 0.045), after PNO at 10 mins. (96.94 $\pm 13.00 \mathrm{bpm}$ vs $89.24 \pm$ $14.19 \mathrm{bpm} ; \mathrm{P}=0.006)$, at 20 mins. (98.08 $\pm 13.46 \mathrm{bpm}$ vs $90.34 \pm 15.10 \mathrm{bpm} ; \mathrm{P}=0.008)$, and at 30 mins. (94.7 \pm 11.42 bpm vs $88.72 \pm 14.51 \mathrm{bpm} ; \mathrm{P}=0.024$ ).

In another study, ${ }^{9}$ significant rise in MAP was observed in control group compared to clonidine group ( $8 \mathrm{mcg} / \mathrm{kg}$ ) (Preinduction; $98 \mathrm{mmHg}$ vs $75 \mathrm{mmHg}$ ), at (15 mins. after PNO; 90 vs $72 \mathrm{mmHg}$ ), at (30 mins. $89 \mathrm{mmHg}$ vs $70 \mathrm{mmHg}):(\mathrm{p}<$ 0.001). In our study also mean MAP raised significantly in Group S as compared to Group C, that is before PNO (108.90 $\pm 7.57 \mathrm{mmHg}$ vs $103.26 \pm 10.22 \mathrm{mmHg}$; $\mathrm{p}=0.002$ ), after PNO at 10 mins. $(115.98 \pm 9.79 \mathrm{mmHg}$ vs $105.64 \pm 9.94$ $\mathrm{mmHg} ; \mathrm{p}<0.001)$, at 20 mins. $(116.40 \pm 8.05 \mathrm{mmHg}$ vs $106.96 \pm 9.53 \mathrm{mmHg} ; \mathrm{p}<0.001)$, and at $30 \mathrm{mins}$. $(113.08 \pm 7.13$ mmHg vs $104.40 \pm 9.92 \mathrm{mmHg}$; $<0.001$ ).

In the same study, significant rise in HR was observed in control group compared to clonidine group (at pre-induction $74 \mathrm{bpm}$ vs $66 \mathrm{bpm}$ ), (at 15 mins. after PNO; $76 \mathrm{bpm}$ vs 63 bpm) and at (30 mins.; $89 \mathrm{bpm}$ vs $7 \mathrm{p} \mathrm{bpm).} \mathrm{In} \mathrm{our} \mathrm{study} \mathrm{also}$ mean HR was significantly raised in Group $S$, that is before PNO (91.96 $\pm 12.36 \mathrm{bpm}$ vs $86.92 \pm 12.46 \mathrm{bpm}$; $\mathrm{P}=0.045)$, after PNO at 10 mins. $(96.94 \pm 13.00 \mathrm{bpm}$ vs $89.24 \pm 14.19 \mathrm{bpm}$; $\mathrm{P}=$ $0.006)$, at 20 mins. (98.08 $\pm 13.46 \mathrm{bpm}$ vs $90.34 \pm 15.10 \mathrm{bpm}$; $\mathrm{P}=0.008)$, and at 30 mins. $(94.7 \pm 11.42 \mathrm{bpm}$ vs $88.72 \pm 14.51$ bpm; $\mathrm{P}=0.024)$.

In the present study, VAS scores were significantly lower in clonidine group in the first 12 hours, 5 to 15 mins. $3.00 \pm$ $00(\mathrm{P}=0.000), 15$ mins. to 2 hrs. $3.48 \pm 0.505(\mathrm{P}=0.004), 2$ hrs. to 4 hrs. $4.04 \pm 0.786$ ( $\mathrm{P}=0.000), 4$ hrs. to $12 \mathrm{hrs}$. $3.40 \pm$ $0.495(\mathrm{P}=0.549)$.

Up to $4 \mathrm{hrs}$. post-operatively, VAS scores were significantly lower without the use of analgesic for the first 4 hrs. compared to control group with the use of diclofenac sodium as analgesic. These findings suggest that clonidine group of patients did not require analgesic for up to $4 \mathrm{hrs}$. P.O.

A study 12 where patients were pre-medicated with clonidine $150 \mathrm{mcg}$ orally displayed greater haemodynamic stability perioperatively with VAS scores statistically significant and the postoperative analgesic requirement was significantly less $(1.5 \pm 1.3$ vs $2.2 \pm 1.3)$ dose and the time for the first dose of analgesic was prolonged ( $411 \pm 565$ vs $264 \pm$ 441 mins.) in clonidine group $\mathrm{p}<0.005$, whereas in our study VAS scores were significantly lower in Group C with statistical significance $p<0.001$ up to $12 \mathrm{hrs}$. and the time for first analgesic was prolonged up to a minute postoperatively $(\mathrm{p}<0.001)$.

A study ${ }^{13}$ to assess the effects of clonidine $100 \mathrm{mcg}$ on postoperative pain and morphine consumption after abdominal hysterectomy showed that total morphine consumption and VAS scores were lower in clonidine group up to $48 \mathrm{hrs}$. compared to control group and morphine consumption was less in clonidine group $20 \pm 1.28$ vs $26.9 \pm$ 2.8 ( $\mathrm{p}<0.05$ ); comparable to our study VAS scores were significantly lower in Group C with ( ) up to $12 \mathrm{hrs}$. and up to 24 hrs. and time for first postoperative analgesic was prolonged up to a minute post-operatively.

In another study, ${ }^{14}$ twenty ASA physical status 2 or 3 patients recovering from abdominal surgery were allocated randomly to receive either epidural fentanyl (100 micrograms in $10 \mathrm{~mL}$ isotonic saline; $\mathrm{EF}$ group) or epidural fentanyl (same dose) plus epidural clonidine (150 micrograms; EF + C group) in isotonic saline solution. Pain was evaluated at 15 mins. for 2 hours and 30 mins. for 4 hours. Maximum pain relief was $68.2(24.1 \%)$ of initial VAS scores in clonidine group compared to $14.7(25.2 \%)$ in saline group and duration of analgesia was $210 \pm 87$ mins. in clonidine group compared to $45 \pm 27$ mins. in saline group. In our study, VAS scores were significantly lower up to $12 \mathrm{hrs}$. with statistical significance $(\mathrm{p}<0.001)$.

Overall, the present study showed that administration of IV clonidine at $1.5 \mu \mathrm{cg} / \mathrm{kg}$ as premedication in patients undergoing laparoscopic cholecystectomy provides stable perioperative haemodynamics, protection against adverse haemodynamic response triggered by pneumoperitoneum reduction in post-operative pain and requirement of analgesic.

\section{CONCLUSION}

From the results of the present study following conclusion may be drawn with the use of intravenous clonidine at 1.5 $\mu \mathrm{cg} / \mathrm{kg}$ as premedication.

1. Maintains heart rate during pneumoperitoneum.

2. Mean arterial blood pressure is maintained with clonidine as it gives more haemodynamic stability.

3. Reduces VAS scores and prolongs the requirement of first post-operative analgesic.

\section{REFERENCES}

[1] Vecchio R, MacFayden BV, Palazzo F. History of laparoscopic surgery. Panminerva Med 2000;42(1):87-90.

[2] Hodgson C, McClelland RM, Newton JR. Some effects of peritoneal insufflation of carbon dioxide at laparoscopy. Anaesthesia 1970;25(3):382-90.

[3] Antony C, Noreen D. Intraabdominal laparoscopic surgery: anaesthetic implication in wylie and Churchill Davidsons. A practice of anesthesia. 7th edn. London; Hodder Arnold Publications 2003.

[4] Koivusalo AM, Lindgren L. Effects of $\mathrm{CO} 2$ pneumoperitoneum for laparoscopic cholecystectomy. Acta Anaesthesiol Scand 2000;44(7):834-41.

[5] Joris JL, Noirot DP, Legrand MJ, et al. Hemodynamic changes during laparoscopic cholecystectomy. Anaesth Analg 1993;76(5):1067-72. 
[6] Karayiannakis AJ, Makri GG, Mantzioka A, et al. Systemic stress response after laparoscopic or open cholecystectomy: a randomized trial. $\mathrm{Br} \mathrm{J}$ Surg 1997;84(4):467-71.

[7] Ghignone M, Calvillo O, Quintin L. Anaesthesia \& hypertension: the effect of clonidine on perioperative hemodynamics \& isoflurane requirements. Anaesthesiology 1987;67(1):3-10.

[8] Quintin L, Duke PC, Kehler CH, et al. Effect of clonidine on narcotic requirement \& hemodynamic response during induction of fentanyl anaesthesia \& endotracheal intubation. Anaesthesiology 1986;64(1):36-42.

[9] Joris J, Chinche JD, Lamy M. Clonidine reduced hemodynamic changes induced by pneumperitoneum during laparoscopic cholecystectomy. $\mathrm{Br} \quad \mathrm{J}$ Anaesthesiology 1995;74(suppl):A124.

[10] Sung CS, Lin SH, Chan KH, et al. Effect of oral clonidine premedication on perioperative hemodynamic response \& post-operative analgesic requirement for patients undergoing laparoscopic cholecystectomy. Acta Anaesthesiol Sin 2000;38(1):23-9.
[11] Ruffolo R. Physiology and biochemistry of the peripheral autonomic nervous system. In: Wingard L, Brody T, Larner J, et al. (edtr). Human Pharmacology: Molecular-to-Clinical, St. Louis: Mosby-Year Book; 1991:77.

[12] Farquhar-Smith WP. Anatomy, physiology and pharmacology of pain. Anaesthesia and Intensive Care Medicine 2008;9(1):3-7.

[13] Joris JL, Chiche JD, Canivet JL, et al. Haemodynamic changes induced by laparoscopy and their endocrine correlates: effects of clonidine. J Am Coll Cardiol 1998;32(5):1389-96.

[14] Odeberg S, Ljungqvist 0 , Svenberg $\mathrm{T}$, et al. Haemodynamic effects of pneumoperitoneum and the influence of posture during anaesthesia for laparoscopic surgery. Acta Anaesthesiol Scand 1994;38(3):276-83. 